\title{
Universal Velocity Distribution for Smooth and Rough Open Channel Flows
}

\author{
J. H. Pu \\ Lecturer,University of Southampton (Malaysia Campus),No. 3 Persiaran Canselor 1,Kota Ilmu, Educity@Iskandar, \\ 79200 Nusajaya, Malaysia. \\ J.H.Pu@soton.ac.uk
}

(Received December 21, 2011; accepted March 25, 2012)

\begin{abstract}
The Prandtl second kind of secondary current occurs in any narrow channel flow causing velocity dip in the flow velocity distribution by introducing the anisotropic turbulence into the flow. Here, a study was conducted to explain the occurrence of the secondary current in the outer region of flow velocity distribution using a universal expression. Started from the basic Navier-Stokes equation, the velocity profile derivation was accomplished in a universal way for both smooth and rough open channel flows. However, the outcome of the derived theoretical equation shows that the smooth and rough bed flows give different boundary conditions due to the different formation of log law for smooth and rough bed cases in the inner region of velocity distribution. Detailed comparison with a wide range of different measurement results from literatures (from smooth, rough and field measured data) evidences the capability of the proposed law to represent flow under all bed roughness conditions.
\end{abstract}

Keywords: Universal velocity profile, Secondary current, Velocity dip, Smooth bed flow, Rough bed flow, Open channel flows

\section{NOMENCLATURE}

\begin{tabular}{|c|c|c|c|}
\hline$A_{r}$ & aspect ratio $(=b / h)$ & $t$ & time \\
\hline$b$ & free surface flow width & $\tau$ & shear stress \\
\hline $\begin{array}{l}B_{r} \\
h\end{array}$ & $\begin{array}{l}\text { logarithmic integration constant } \\
\text { water depth }\end{array}$ & $U, V, W$ & $\begin{array}{l}\text { velocity in } \mathrm{x}, \mathrm{y} \text { and } \mathrm{z} \text { directions } \\
\text { respectively }\end{array}$ \\
\hline$h_{\max }$ & maximum water depth & $u^{\prime}, v^{\prime}, w^{\prime}$ & turbulent velocity fluctuation in $\mathrm{x}$, \\
\hline $\begin{array}{l}k_{s} \\
\kappa\end{array}$ & $\begin{array}{l}\text { Nikuradse roughness } \\
\text { von Karman constant }\end{array}$ & $u_{*}$ & $\begin{array}{l}\mathrm{y} \text { and } \mathrm{z} \text { directions respectively } \\
\text { shear velocity }\end{array}$ \\
\hline K & kinematic viscosity & $x, y, z$ & $\begin{array}{l}\text { streamwise, lateral and vertical } \\
\text { directions/locations }\end{array}$ \\
\hline$v_{t}$ & turbulent kinematic viscosity & $z_{o}$ & reference 'zero-velocity' depth \\
\hline$P$ & pressure & & near to bed \\
\hline$\Pi$ & wake parameter & $\xi$ & $z / h$ \\
\hline$\rho$ & density & $\xi_{1}$ & $\xi$ at inner region boundary of \\
\hline$S_{o}$ & channel bed slope & $\xi_{d i p}$ & $\begin{array}{l}\text { velocity distribution } \\
\xi \text { at velocity dip position }\end{array}$ \\
\hline
\end{tabular}

\section{INTRODUCTION}

In the light of literatures, the velocity distribution of any open channel flow is dictated by the aspect ratio, $A_{r}$, of the channel. For the velocity investigation of a flow, logarithmic-based laws are commonly used to represent the measured velocity data. In this method, the measured velocity is normalised using the shear or maximum velocity, and the normalised velocity is then represented in a logarithmic profile. The law of wall, first suggested by Keulegan (1938) and Nikuradse (1950), is one of the earliest logarithmic methods in representing the normalised velocity profile. This method is commonly believed to be insufficient to represent the outer region of the velocity profile. Coles (1956) proposed an improved law of wake to represent the flow velocity, and it is proven to more accurately represent the normalised flow velocity profile in the 
outer region compared to the $\log$ law (see Kironoto and Graf, 1994, Song and Graf, 1996).

In recent years, a variety of laws for turbulent velocity profile were suggested in literatures for either smooth or rough bed flow (e.g. Guo and Julien, 2003, Yang et al. 2005, Termini and Greco, 2006, Liu and Ni, 2007, Guo and Julien, 2008). Majority of them utilized the modification of $\log$ or wake law to represent their velocity profiles. However, there are two factors that are often assumed: 1) the modified laws often only representative for either smooth or rough bed flow, and 2 ) the velocity-dip phenomenon observed in the narrow or natural channels are often neglected.

Wang and Cheng $(2005,2006)$ measured the secondary current created by artificial bed, which consists of smooth and rough strips. In their studies, the modified log law was used to represent the velocity distribution with the occurrence of dip phenomenon. In Yang et al. (2006) investigation of velocity dip, the gradually accelerating flow was used. Both of their studies emphasised the importance of velocity dip consideration in estimating the velocity profile under the influence of secondary current.

To improve the common short-coming for most of the proposed laws from literatures, in this paper, a universal law was proposed to represent both smooth and rough open channel flows. The proposed law in this paper was developed using the assumption of the fully turbulent flow, hence, the secondary current effect was not ignored like the common laws found in literatures. The proposed law has also been tested against the measurement data from literatures with various conditions to investigate its capability.

\section{THEORETICAL DEVELOPMENT}

To derive the velocity profile, the well-known NavierStokes equation is used. It reads

$\frac{\partial U_{i}}{\partial t}+U_{j} \frac{\partial U_{i}}{\partial x_{j}}=-\frac{1}{\rho} \frac{\partial P}{\partial x_{i}}+v \frac{\partial^{2} U_{i}}{\partial x_{k} \partial x_{k}}-\frac{\partial}{\partial x_{j}}\left(\overline{u_{i} u_{j}}\right)+g_{i}$

where $U_{i}$ is the mean velocity in the $i$-direction ( $i=1,2,3$ in the streamwise $x$, lateral $y$, and vertical $z$ directions respectively), $\rho$ is the fluid density, $P$ is the distributed pressure, $v$ is the fluid kinematics viscosity, $\overline{\left(u_{i} u_{j}\right)}$ are components of the Reynolds stress tensor, $g$ is the gravitational acceleration, and $t$ denotes the time domain. Thus, the steady, uniform and fully developed turbulent momentum equation in the streamwise direction could be written as

$V \frac{\partial U}{\partial y}+W \frac{\partial U}{\partial z}=g \sin \theta+\frac{\partial(-\overline{u v})}{\partial y}+\frac{\partial(-\overline{u w})}{\partial z}+v \nabla^{2} U$

where $U, V$ and $W$ are velocities in $x, y$ and $z$ directions respectively, $\sin \theta$ is the gravitational parameter of $g$, and $v$ is the kinematic viscosity. From the derivation above, the integration of Eq. (2) in the vertical direction from the interval of vertical point, $z$, to full water depth, $h$, yields
$\left.U W\right|_{h}-\left.U W\right|_{z}=g(h-z) \sin \theta+\frac{\left(\left.\tau\right|_{h}-\left.\tau\right|_{z}\right)}{\rho}$

in which, shear stress $\tau$, could be written as

$\frac{\tau}{\rho}=v \frac{\partial U}{\partial z}-\overline{u w}$

At the free surface of any open channel, the vertical differentiate term, the vertical velocity, and its velocity fluctuation is ignorable. By using those definitions into Eq. (3), one could obtain

$\frac{\tau}{\rho}-U W=\alpha\left(1-\frac{z}{h}\right)$

where $\alpha=g h \sin \theta$. From Rodi (1993), we know that Boussinesq's equation could be written in a form of

$-\overline{u w}=v_{t} \frac{\partial U}{\partial z}$

and, turbulent kinematic viscosity $v_{t}$ could be written as (Yang et al. 2004)

$v_{t}=u_{*} \kappa z\left(1-\frac{z}{h}\right)$

In Eq. (7), $u_{*}$ is the shear velocity, and $\kappa$ is the von Karman constant. Combining Eq. (4)-(6) and Eq. (7) into Eq. (5), we could get

$\frac{\partial U}{\partial \xi}+\left(\frac{-W}{\left[v / h+u_{*} \kappa \xi(1-\xi)\right]}\right) \cdot U=\frac{\alpha(1-\xi)}{\left[v / h+u_{*} \kappa \xi(1-\xi)\right]}$

where $\xi=z / h$.

\section{Analytical Analysis AND SIMPLIFICATION}

Using the complicated differential form of Eq. (8), the streamwise velocity could not be solved. To find the full solution of $U$, we can integrate Eq. (8) to break the differential equation into a first order equation form. By regrouping Eq. (8), we will get

$\frac{d U(\xi)}{d \xi}+F(\xi) U(\xi)=G(\xi)$

By comparing Eq. (8) to Eq. (9), one can obtain

$F(\xi)=\frac{-W(\xi)}{\left[v / h+u_{*} \kappa \xi(1-\xi)\right]}$
$G(\xi)=\frac{\alpha \cdot(1-\xi)}{\left[v / h+u_{*} \kappa \xi \cdot(1-\xi)\right]}$

An intermediate general solution function of Eq. (9) can be formed as

$I(\xi)=\exp \left(\int_{\xi_{1}}^{\xi} F(\xi) d \xi\right)$

where, at constant inner region boundary $\xi_{1}$, the integration goes to 0 , and it gives $I\left(\xi_{1}\right)=1$. Combining Eq. (11) into Eq. (9), we will have 
$\frac{d[I(\xi) \cdot U(\xi)]}{d \xi}=I(\xi) G(\xi)$

Further integrates Eq. (12) through $\xi_{1} \rightarrow \xi$ gives

$$
U(\xi)=I^{-1}(\xi)\left[\int_{\xi_{1}}^{\xi} I(\xi) G(\xi) d \xi+U\left(\xi_{1}\right)\right]
$$

As showing in Eq. (8), an expression for $W$ is needed to complete the solution of Eq. (13). The suggested $W$ from Bonakdari (2006), and Nezu and Nakagawa (1993) are investigated in this study. From Bonakdari (2006), the flow normalized vertical velocity could be expressed as follows

$\frac{W}{u_{*}}=\frac{-P_{A} \kappa \xi\left(1-\xi^{2}\right)}{\xi^{2} / 2+\xi+C_{A r}}$

where, $P_{A}$ and $C_{A r}$ are the empirical constants used for the $W / u_{*}$ profile fitting. Equation (14a) has been further simplified by Bonakdari et al. (2008) as testing shows that unity $P_{A}$ gives good correspondence in representing the streamwise velocity distribution.

$\frac{W}{u_{*}}=\frac{-\kappa \xi\left(1-\xi^{2}\right)}{\xi^{2} / 2+\xi+C_{A r}}$

Using the suggestion by Ikeda (1981) and Nezu and Nakagawa (1993), $W$ profile could be formulate as follows

$\frac{W}{u_{*}}=-\frac{6 \alpha_{b}}{\kappa \pi^{2}} \cos \left(\frac{\pi y}{h}\right)[(2 \xi-1) \cos (\pi \xi)+1]$

where $\alpha_{b}$ is the amplitude of the perturbation at bed Reynolds stress $\tau_{b}$. Hence, at the centre of the channel, Eq. (15a) could be reduced to

$\frac{W}{u_{*}}=-\frac{6 \alpha_{b}}{\kappa \pi^{2}}[(2 \xi-1) \cos (\pi \xi)+1]$

In Fig. 1, the prediction using Eq. (14b) is compared to the relation suggested by Ikeda (1981) and Nezu and Nakagawa (1993) in Eq. (15a). The results also compared to the experimental data from $\mathrm{Pu}$ (2008) in the same figure. One could observe that the experimental measurements by $\mathrm{Pu}$ (2008) on both rough and smooth bed flow tests collapse well with the theoretical equations suggested by both Ikeda (1981), and Bonakdari (2006). $\alpha_{b}$ used in Eq. (15b) for the comparison falls between the proposed value by Nezu and Nakagawa (1993) - 0.10, and Ikeda (1981) - 0.20. Comparatively, Bonakdari's Eq. (14b), which has been adopted into this study, gives a better prediction to the experimental measurements. Furthermore, the theoretical equation also well represents vertical velocity of a flow regardless the bed roughness condition.

In the argument of Bonakdari et al. (2008) together with $\mathrm{Pu}$ et al. (2010), the representation of $W$ by Eq. (14b) is sufficient to be used in the streamwise velocity profile derivation. Hence in our consideration, Eq. (14b) will be employed instead of Eq. (14a). It is because Eq. (14b), which consists only 1 degree of adjustment by $C_{A r}$, promotes the confident in the streamwise velocity profile estimation if compared to Eq. (14a), which consists 2 degree of adjustment, $P_{A}$ and $C_{A r}$.

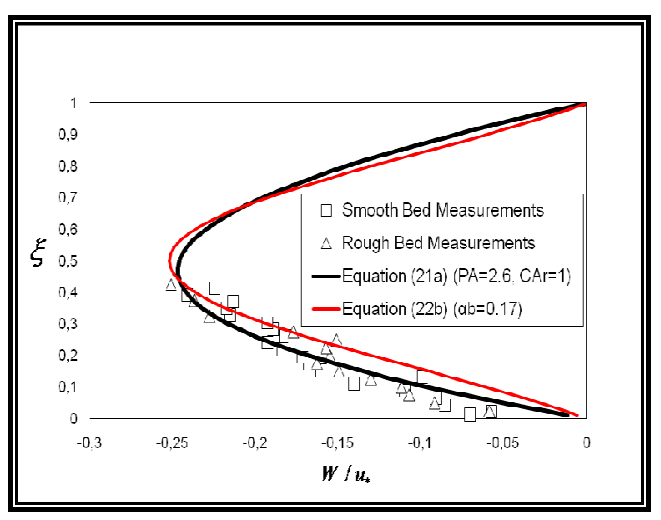

Fig. 1. Normalized vertical velocity profiles comparison.

By using the expression of $W$ in Eq. (14b) into Eq. (11), we could obtain the following

$$
\begin{aligned}
& I(\xi)=\exp \left(\int_{\xi_{1}}^{\xi} \frac{-W(\xi)}{\left[v / h+u_{*} \kappa \xi(1-\xi)\right]} d \xi\right) \\
& =\exp \left(\int_{\xi_{1}}^{\xi} \frac{\kappa u_{*} \xi h\left(1-\xi^{2}\right)}{\left(\xi^{2} / 2+\xi+C_{A r}\right)\left[v+u_{*} \kappa \xi h(1-\xi)\right]} d \xi\right)
\end{aligned}
$$

Tests on three separate results from Hurther and Lemmin (2001), Nezu and Azuma (2004), and Storm and Papanicolaou (2007) have been conducted to compare the difference of domain for the integrated fraction at Eq. (16) - comparing $v+u_{*} \kappa \xi h(1-\xi)$ with results from simply $u_{*} \kappa \xi h(1-\xi)$. The comparison results are showing in Fig. 2 to Fig. 4.

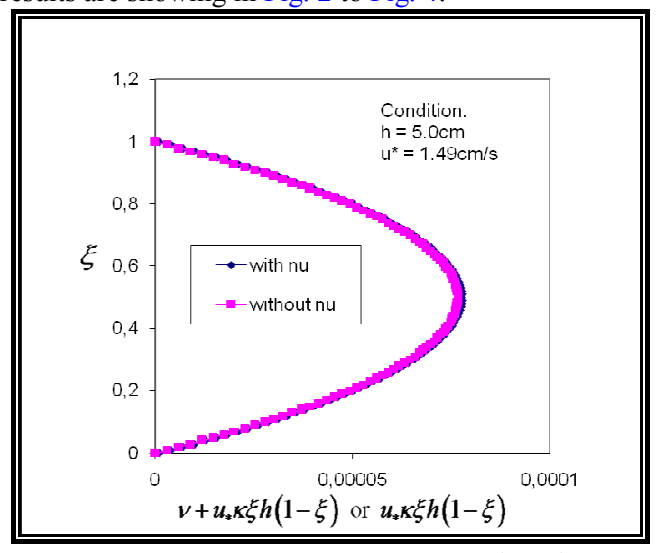

Fig. 2. Comparison of $v+u_{*} \kappa \xi h(1-\xi)$ with $u_{*} \kappa \xi h(1-\xi)$ [smooth bed experimental flow on rectangular channel by Nezu and Azuma (2004)]

The three tests consist of a smooth bed flow (Nezu and Azuma 2004), a rough bed flow (Hurther and Lemmin, 2001) and a field case study (Storm and Papanicolaou, 
2007) to conduct a thorough investigation. $L_{2}$ norm analysis is also presented for the comparison results (shown in Fig. 5). The equation of $L_{2}$ used is presented as below

$L_{2}=\sqrt{\frac{\sum_{\xi}\left(F_{\xi}^{w n}-F_{\xi}^{n}\right)^{2}}{\sum_{\xi}\left(F_{\xi}^{n}\right)^{2}}}$

where, $F_{\xi}^{w n}$ and $F_{\xi}^{n}$ are $u_{*} \kappa \xi h(1-\xi)$ (without $v$ ) and $v+u_{*} \kappa \xi h(1-\xi)$ (with $v$ ) functions respectively.

The $L_{2}$ analysis is done at the basic of $\xi$ step.

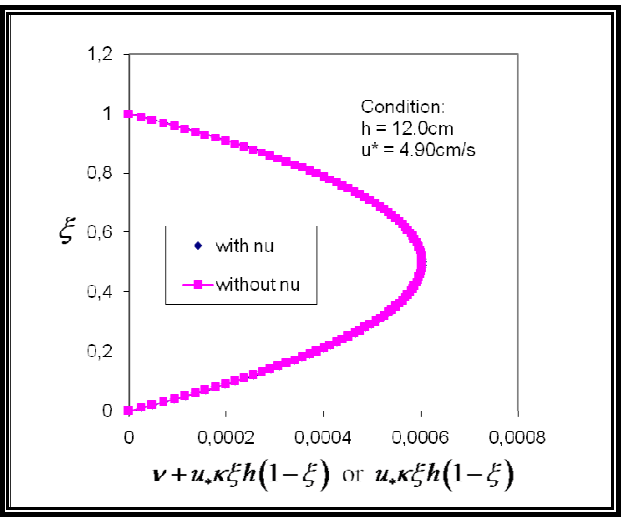

Fig. 3. Comparison of $v+u_{*} \kappa \xi h(1-\xi)$ with $u_{*} \kappa \xi h(1-\xi)$ [rough bed experimental flow on rectangular channel by Hurther and Lemmin (2001)]

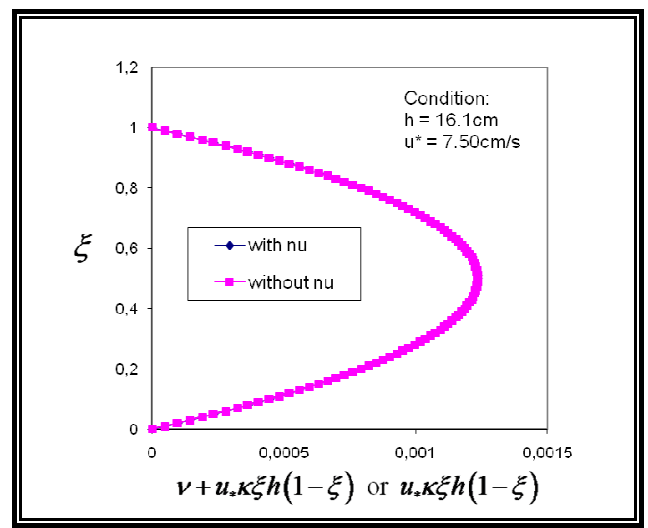

Fig. 4. Comparison of $v+u_{*} \kappa \xi h(1-\xi)$ with $u_{*} \kappa \xi h(1-\xi)$ [field study measurements on river by Storm and Papanicolaou (2007)]

Both fraction domain and $L_{2}$ norm analyses demonstrate that $v+u_{*} \kappa \xi h(1-\xi) \approx u_{*} \kappa \xi h(1-\xi)$, since $u_{*} \kappa \xi h(1-\xi) \gg v$ (Fig. 2 to Fig. 5). Also from $L_{2}$ norm analysis in Fig. (5a) conclusion could be drawn rough bed flow with higher $h$ gives better assumption of $v+u_{*} \kappa \xi h(1-\xi) \approx u_{*} \kappa \xi h(1-\xi)$ compared to the smooth bed low- $h$ flow. By employing the outcome of the test in Fig. 2 to Fig. 5, we could reduce Eq. (17) to

$$
\begin{aligned}
& I(\xi)=\exp \left(\int_{\xi_{1}}^{\xi} \frac{u_{*} \kappa \xi h\left(1-\xi^{2}\right)}{\left(\xi^{2} / 2+\xi+C_{A r}\right)\left[u_{*} \kappa \xi h(1-\xi)\right]} d \xi\right) \\
& =\left[\frac{\xi^{2} / 2+\xi+C_{A r}}{\xi_{1}^{2} / 2+\xi_{1}+C_{A r}}\right]
\end{aligned}
$$

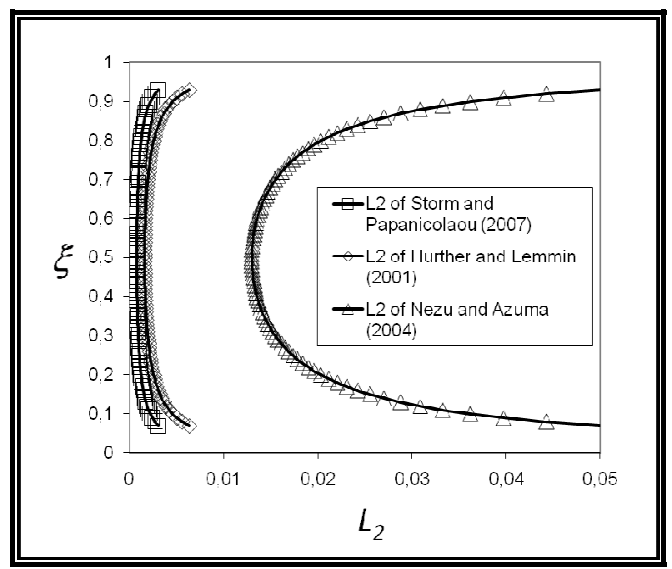

Fig. 5. $L_{2}$ analysis of comparison between

$$
v+u_{*} \kappa \xi h(1-\xi) \text { with } u_{*} \kappa \xi h(1-\xi)
$$

From Eq. (18), one could obtain

$\int_{\xi_{1}}^{\xi} I(\xi) G(\xi) d \xi$

$$
\begin{aligned}
& =\int_{\xi_{1}}^{\xi} \frac{\alpha(1-\xi)}{\left[v / h+u_{*} \kappa \xi(1-\xi)\right]}\left[\frac{\xi^{2} / 2+\xi+C_{A r}}{\xi_{1}^{2} / 2+\xi_{1}+C_{A r}}\right] d \xi \\
& =\frac{\alpha\left[\left(\xi^{2} / 4+\xi\right)-\left(\xi_{1}^{2} / 4+\xi_{1}\right)+C_{A r} \ln \left(\xi / \xi_{1}\right)\right]}{h u_{*} \kappa\left(\xi_{1}^{2} / 2+\xi_{1}+C_{A r}\right)}
\end{aligned}
$$

Substituting Eq. (18) and Eq. (19) into Eq. (13), we could finally get

$$
\begin{aligned}
& U(\xi)=\left[\frac{\xi_{1}^{2} / 2+\xi_{1}+C_{A r}}{\xi^{2} / 2+\xi+C_{A r}}\right] . \\
& {\left[\frac{\alpha\left[\left(\xi^{2} / 4+\xi\right)-\left(\xi_{1}^{2} / 4+\xi_{1}\right)+C_{A r} \ln \left(\xi / \xi_{1}\right)\right]}{h u_{*} \kappa\left(\xi_{1}^{2} / 2+\xi_{1}+C_{A r}\right)}+U\left(\xi_{1}\right)\right]}
\end{aligned}
$$

Equation (20) shows the $U$ profile could be purely expressed in the form of $\xi$. One could observe that Eq. (20) has not utilised the channel roughness hence it is applicable for both smooth and rough open channel flows. This characteristic gives a strategic advantage to the proposed law from any modified log or wake laws usually proposed in literature, since it eases the application process and widening the application capability

\section{BOUNDARY CONSIDERATION}

The As a well-known fact, the log law is capable to represent the inner region of a flow velocity profile. In 
order to determine the boundary $U\left(\xi_{1}\right)$ in Eq. (20), the $\log$ law is used in this study, which it takes the forms of

Smooth Bed:

$\frac{U}{u_{*}}=\frac{1}{\kappa} \ln \left(\frac{z u_{*}}{v}\right)+B_{r}$

Rough Bed:

$\frac{U}{u_{*}}=\frac{1}{\kappa} \ln \left(\frac{z-z_{o}}{k_{s}}\right)+B_{r}$

where, $z_{o}$ is the reference 'zero-velocity' depth near to

bed, and $k_{s}$ is the Nikuradse roughness. By fitting the

$U\left(\xi_{1}\right)$ boundaries into Eq. (20), we get the normalised velocity profile as

Smooth Bed:

$\frac{U}{u_{*}}=\left[\frac{\xi_{1}^{2} / 2+\xi_{1}+C_{A r}}{\xi^{2} / 2+\xi+C_{A r}}\right]$.

$\left[\frac{\alpha\left[\left(\xi^{2} / 4+\xi\right)-\left(\xi_{1}^{2} / 4+\xi_{1}\right)+C_{A r} \ln \left(\xi / \xi_{1}\right)\right]}{h u_{*}^{2} \kappa\left(\xi_{1}^{2} / 2+\xi_{1}+C_{A r}\right)}+\frac{1}{\kappa} \ln \left(\frac{\xi_{h} h u_{*}}{v}\right)+B_{r}\right]$
Rough Bed:

$\frac{U}{u_{*}}=\left[\frac{\xi_{1}^{2} / 2+\xi_{1}+C_{A r}}{\xi^{2} / 2+\xi+C_{A r}}\right]$.

$\left[\frac{\alpha\left[\left(\xi^{2} / 4+\xi\right)-\left(\xi_{1}^{2} / 4+\xi_{1}\right)+C_{A r} \ln \left(\xi / \xi_{1}\right)\right]}{h u_{*}^{2} \kappa\left(\xi_{1}^{2} / 2+\xi_{1}+C_{A r}\right)}+\frac{1}{\kappa} \ln \left(\frac{\xi_{1} h}{k_{s}}\right)+B_{r}\right]$

where, $B_{r}$ is the logarithmic integration constant, in which $B_{r} \approx 4.70$ suggested by $\mathrm{Pu}$ (2008), $B_{r} \approx 4.90$ suggested by Anwar and Atkins (1980) and Mellor and Gibson (1966), and $B_{r} \approx 5.10$ suggested by Coles (1956) and Cardoso et al. (1989) for smooth bed uniform flow studies. For the rough bed uniform flow studies, $B_{r}$ is found to be $8.47 \pm 0.90$ by Kironoto and Graf (1994), $8.42 \pm 0.22$ by Song et al. (1994), $7.80 \pm$ 0.37 by Dey and Raikar (2007), and 6.30 by Pu (2008). In the comparison of different literature studies, the rough bed flow shows higher $B_{r}$ values than the smooth bed flow.

Table 1 Parameters of the referred smooth bed open channel flow measurements from various literatures

\section{CONCLUSION APPlication To SMOOTH OPEN CHANNEL FlOWS}

To examine the validity of the proposed law, comparisons with various published measurements from literature were conducted in this section and the next. The smooth open channel flow prediction is investigated at this section; whereas the next section will discuss the rough open channel flow prediction by the proposed law. All parameters used for the smooth bed flow measurements testing are presented at Table 1. To start the comparison on the smooth channel flow measurements, Sarma et al. (1983) experimental data at region away from bed was used (refer to Fig. 6). In the experiment, a relatively high aspect ratio $A_{r}=6.0$ was set. It was observable that the proposed law gave a better prediction towards the experimental data compared to the famous wake law [shown in Eq. (25) to Eq. (26)] especially near to the region of free surface. The results were also compared to Yang et al. (2004) proposed law for the smooth rectangular channel flow [shown in Eq. (27)]; and better agreement with the measurements was presented by the proposed Eq. (23).

Wake Law:

Smooth Bed:

\begin{tabular}{|c|c|c|c|}
\hline$(\mathrm{m})$ & $\mathrm{u}_{*}(\mathrm{~m} / \mathrm{s})$ & $\mathrm{S}_{\mathrm{o}} \times 10^{-3}(-)$ & Channel \\
\hline .102 & 0.0290 & 0.15 & Wide \\
\hline .092 & 0.0283 & 0.32 & Wide \\
\hline .172 & 0.0410 & 2.00 & Narrow \\
\hline .169 & 0.0410 & 2.00 & Narrow \\
\hline .173 & 0.0410 & 2.00 & Narrow \\
\hline .040 & 0.0387 & 0.20 & Narrow \\
\multicolumn{3}{|c}{$\frac{U}{u_{*}}=\frac{1}{\kappa} \ln \left(\frac{z u_{*}}{v}\right)+B_{r}+\frac{2 \Pi}{\kappa} \sin ^{2}\left(\frac{\pi z}{2 h}\right)$}
\end{tabular}

Rough Bed:

$\frac{U}{u_{*}}=\frac{1}{\kappa} \ln \left(\frac{z-z_{o}}{k_{s}}\right)+B_{r}+\frac{2 \Pi}{\kappa} \sin ^{2}\left(\frac{\pi z}{2 h}\right)$

Yang et al. (2004) Law:

$\frac{U}{u_{*}}=\frac{1}{\kappa} \ln \left(\frac{c_{y} z u_{*}}{v}\right)+\frac{1.3 \exp \left(-0.5 A_{r}\right)}{\kappa} \ln \left(1-\frac{z}{h}\right)$

where, $\Pi$ is the wake parameter and $c_{y}$ is a fitting constant for the log law profile suggested by Yang et al. (2004).

In Fig. 7, the proposed law, Yang et al. (2004) law and the wake law were compared with $\mathrm{Pu}$ (2008) measurements at the mid-water depth region. The proposed law showed closer correspondence to the measurements than both Yang et al. (2004) law and wake law. Due to the high $A_{r}$ in both $\mathrm{Pu}$ (2008) and Sarma et al. (1983) experiments, the velocity dip is not expected to happen. Although the wake law was not predicting the experimental results as accurate as the proposed law; but due to the non-occurrence of the velocity dip, the discrepancy between the proposed law and the wake law was not too great. However, Yang et al. (2004) law clearly ineffective to represent the non- 
secondary current influenced flow near the free surface, as it under-predicted Sarma et al. (1983) velocity profile at the outer region.

Using Coleman (1986) measurements, the narrow channel flow velocity profile (with $2.06 \leq A_{r} \leq 2.11$ )

was tested against the proposed law, Yang et al. (2004) law and the wake law in Fig. 8(a-(c). The proposed law showed a good capability to represent the measured velocity profile closely, except some discrepancies at Run 32; whereas the wake law and Yang et al. (2004) law showed greater discrepancy. In all runs, the log law has reproduced the velocity profile at the inner zone well. In the comparison with Nezu et al. (1985) measurements (Fig. 9), the proposed law has again reproduced a better representation to the measured data than both Yang et al. (2004) law and the wake law in the outer region of the velocity profile.

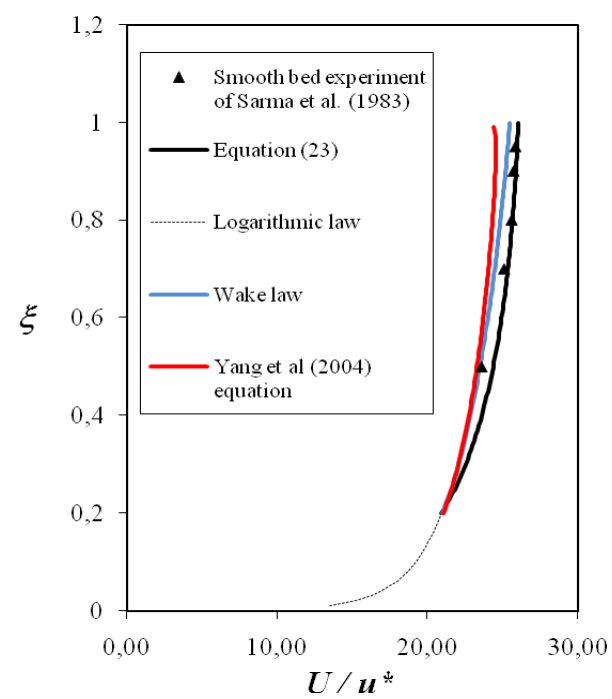

Fig. 6. Laws comparison with the measurements by Sarm et et al. (1983)

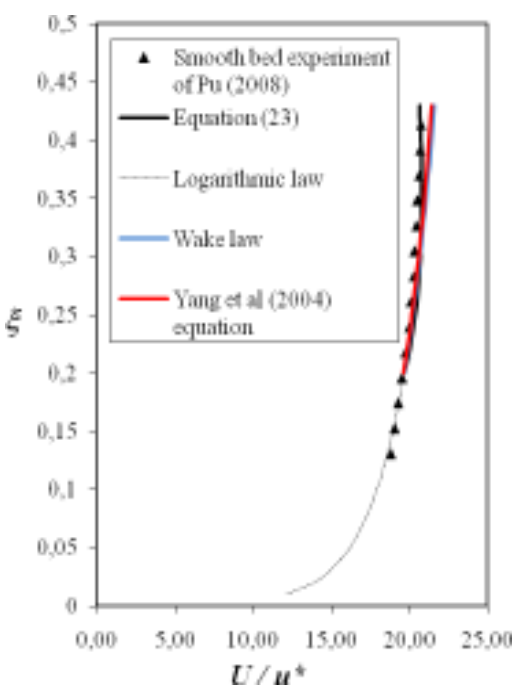

Fig. 7. Laws comparison with the measurements by (Pu 2008)
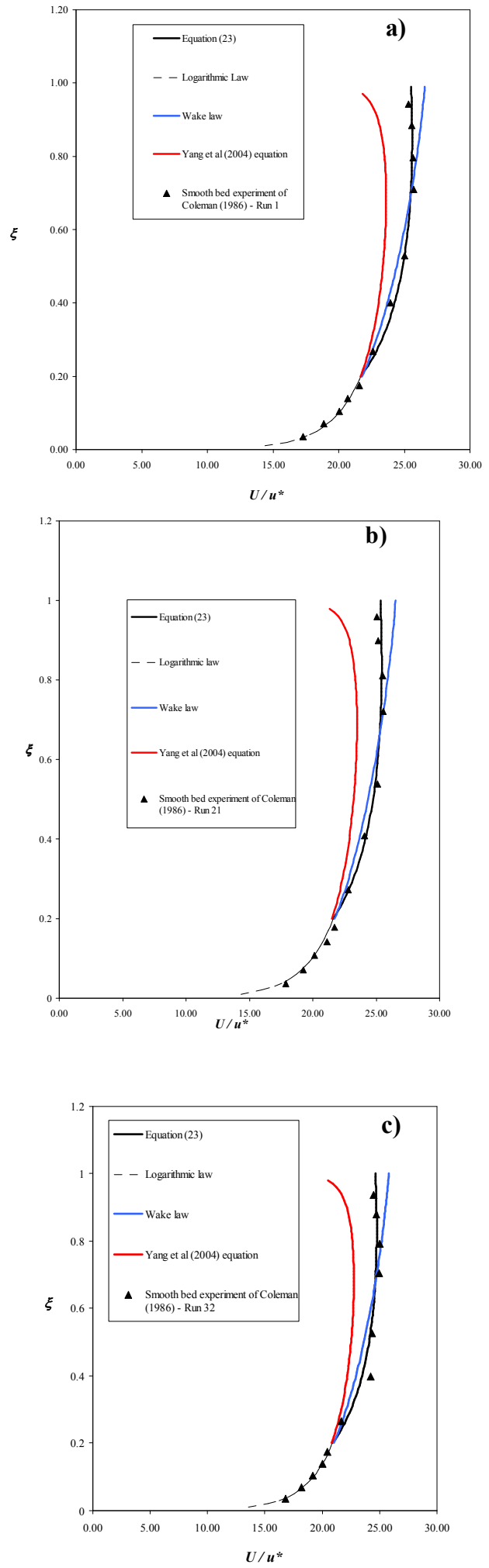

Fig. 8. Laws comparisons with (a) Run 1, (b) Run 21 and (c) Run 32 measurements by Coleman (1986) 


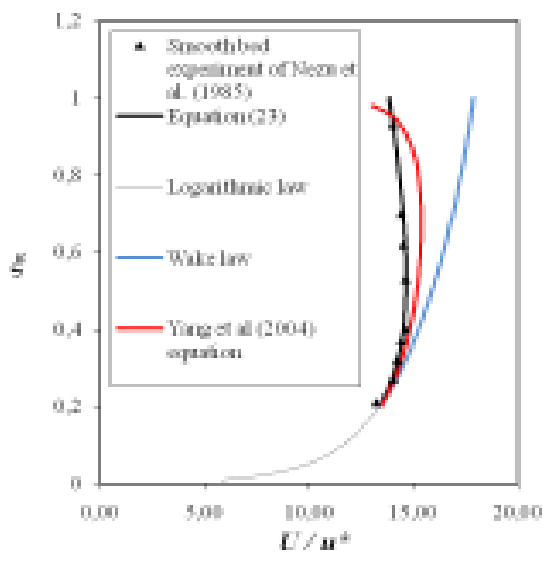

Fig. 9. Laws comparison with the measurements by Nezu et al. (1985)

\section{APPLication To Rough OPEN Channel ANd ACTUAl Field STUdied FLOWS}

In this section, the rough bed flow measurements conducted at channels with different roughness and shapes are used from literature to compare with the proposed law to validate its applicability into wide range of measurements. The parameters used for the rough bed flow measurements testing are presented in Table 2. First, the experimental flow data by Tominaga et al. (1989) at wide rectangular channel was investigated. Figure 10 shows that both the proposed and wake laws predict velocity profile with close correspondence to Tominaga et al. (1989) measurements, since the wide channel flow experiments in Tominaga et al. (1989) has high aspect ratio $\left(A_{r}=7.90\right)$ and the velocity dip did not take place. Larger discrepancy between the proposed law and the wake law was observed at the region near to free surface, where measurements have been restricted and the comparison was inconclusive. However, the velocity distribution predicted by the proposed law at the mid-stream of outer region $(0.2 \leq \xi \leq 0.6)$ presented closer results to the measurements.

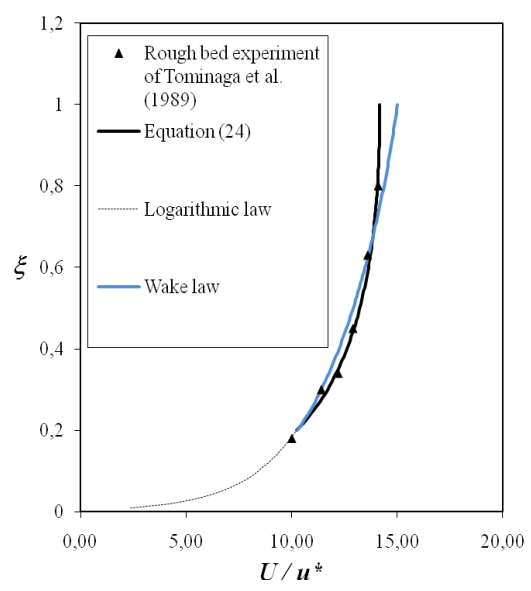

Fig. 10. Laws comparison with the measurements by Tominaga et al. (1989)
To further validate of the proposed law, the narrow channel flow measurements by $\mathrm{Pu}$ (2008) was used for comparison in Fig. 11. In the comparison, the wake law presented high discrepancy in reproducing the velocity distribution; whereas the proposed law captured the measured velocity profile well. In order to examine the validity of the proposed law under the natural channel flow, the comparisons with the actual river measured data by Franca et al. (2008) and Gordon (1992) were conducted. In the comparisons with Franca et al. (2008) data, two separate river site measurements are used from the Venoge and Chamberonne rivers, Vaud, Switzerland. The proposed law estimated the measurements well at Venoge and Chamberonne rivers [Fig. 12(a) and Fig. 12(b) repectively]. The 'S-shaped' velocity distribution shown at Venoge river measurements [Fig. 12(a)], which has been caused by the macro-rough condition in natural river as proposed by Franca (2005), was also well-predicted by the proposed law. In the inner region, the log law reproduced the velocity profile for Venoge and Chamberonne rivers measurements well. Gordon (1992) has measured the velocity profile at the Mississippi river; and it has been used here to compare with the proposed law in Fig. 13. Again, good agreement was presented between the proposed law and the measurements. The comparisons of Gordon (1992) and Franca et al. (2008) field-measured data showed that the proposed law is capable to reproduce the very rough actual river flow velocity profiles, which have not been able to represent precisely by the wake law.

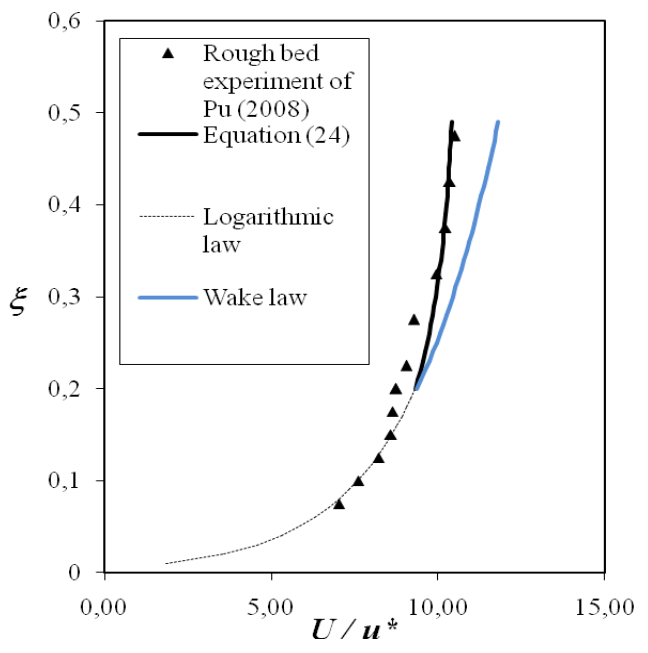

Fig. 11. Laws comparison with the measurements by $\mathrm{Pu}$ (2008)

In the last rough channel flow test, the field measurements of the egg-shaped sewer flow by Larrarte (2006) was used. Four separate measurements with different water depths were employed here for the comparisons. In Fig. 14(a-d), one could observe that the proposed law estimated the measured velocity profile with greater consistency compared to the wake law at all water depth tests. This feature was particularly clear at the near free surface region, where the velocity dip was obvious. 
J. H. Pu / JAFM, Vol. 6, No. 3, pp. 413-423, 2013.

Table 2 Parameters of the referred rough bed open channel flow measurements from literatures.

\begin{tabular}{|c|c|c|c|c|c|c|}
\cline { 2 - 7 } \multicolumn{1}{c|}{} & $\mathrm{A}_{\mathrm{r}}(-)$ & $\mathrm{h}(\mathrm{m})$ & $\mathrm{u} *(\mathrm{~m} / \mathrm{s})$ & $\begin{array}{c}\mathrm{S}_{\mathrm{o}} \mathrm{x} 10^{-3} \\
(-)\end{array}$ & $\mathrm{k}_{\mathrm{s}}(\mathrm{mm})$ & Channel \\
\hline Tominaga et al. $(1989)$ & 7.90 & 0.04 & 0.0348 & 0.20 & 12.0 & Wide \\
\hline $\mathrm{Pu}(2008)$ & 3.75 & 0.12 & 0.0598 & 0.32 & 6.62 & Narrow \\
\hline Franca et al. $(2008)-$ Venoge & 30.00 & 0.21 & 0.0780 & 3.30 & 40.0 & Wide \\
\hline Franca et al. $(2008)-$ Chamberonne & 19.80 & 0.29 & 0.0850 & 2.60 & 49.0 & Wide \\
\hline Gordon $(1992)$ & $>5.00$ & 32.7 & 0.1210 & 0.50 & $\mathrm{z}_{\mathrm{o}}=335$ & Wide \\
\hline Larrarte $(2006)-$ Cerbères $\left(\mathrm{h}_{\max }=0.69 \mathrm{~m}\right)$ & 2.44 & 0.66 & 0.0310 & 0.48 & 1.00 & Narrow \\
\hline Larrarte $(2006)-$ Cerbères $\left(\mathrm{h}_{\max }=0.81 \mathrm{~m}\right)$ & 2.12 & 0.77 & 0.0320 & 0.48 & 0.65 & Narrow \\
\hline Larrarte $(2006)-$ Cerbères $\left(\mathrm{h}_{\max }=1.02 \mathrm{~m}\right)$ & 2.06 & 1.02 & 0.0350 & 0.48 & 0.50 & Narrow \\
\hline Larrarte $(2006)-$ Cerbères $\left(\mathrm{h}_{\max }=1.22 \mathrm{~m}\right)$ & 1.76 & 1.22 & 0.0350 & 0.48 & 0.48 & Narrow \\
\hline
\end{tabular}
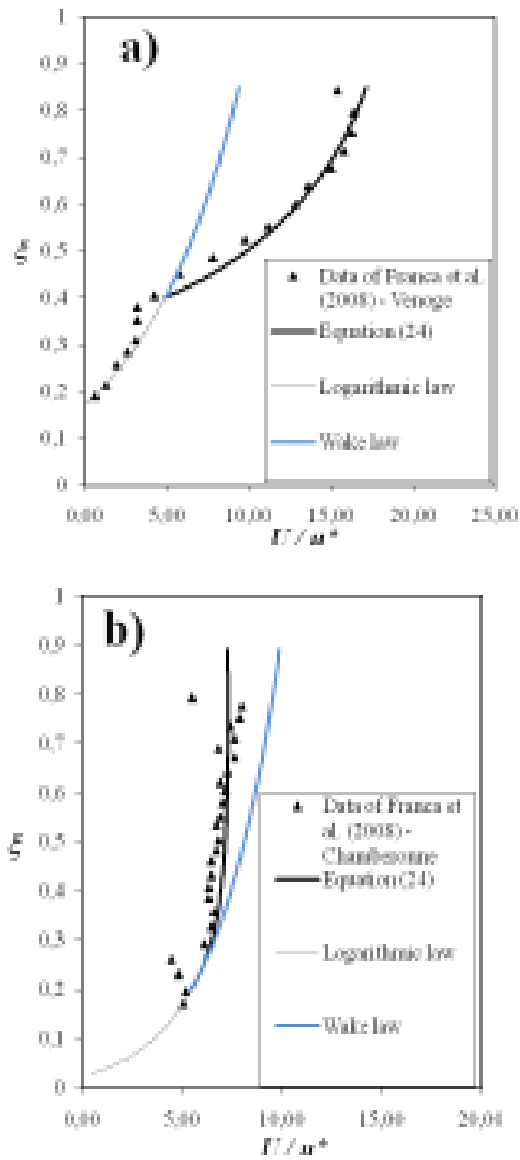

Fig. 12. Laws comparison with a) Venoge, and b) Chamberonne site measurements by Franca et al. (2008)

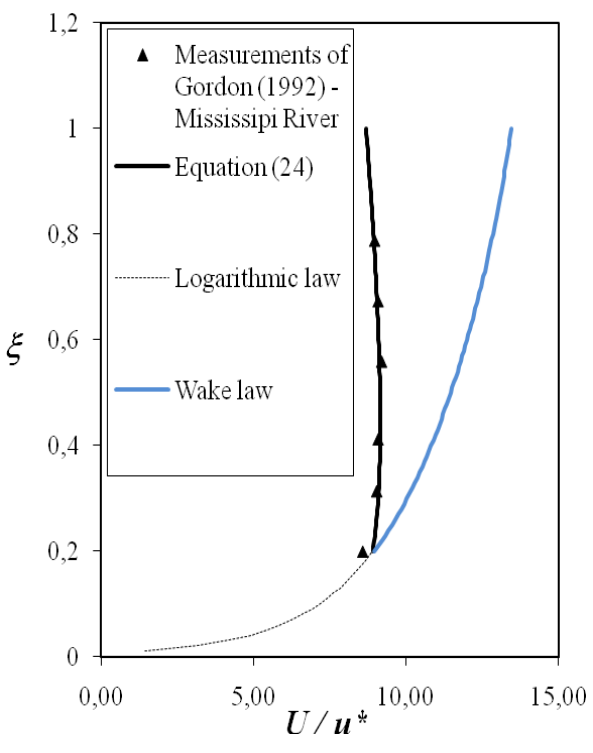

Fig. 13. Laws comparison with the measurements by Gordon (1992)

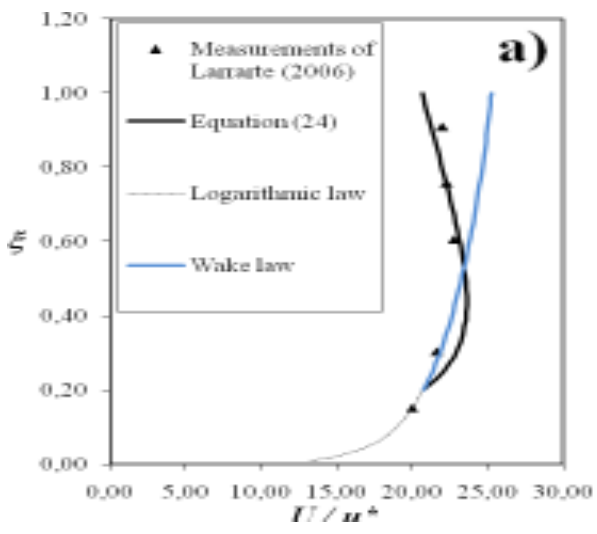



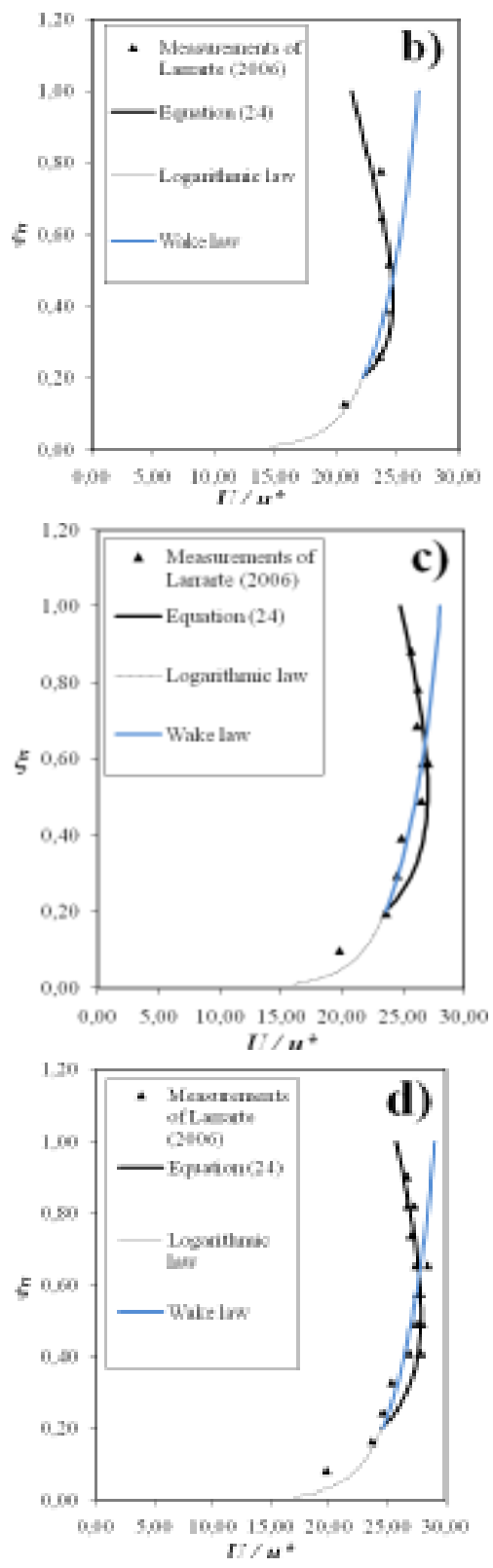

Fig. 14. Laws comparison with the measurements by Larrarte (2006) at Cebères, France, a) $h_{\max }=0.69 m$, b) $\left.h_{\text {max }}=0.81 \mathrm{~m}, \mathrm{c}\right) h_{\text {max }}=1.02 \mathrm{~m}$, and d) $h_{\max }=1.22 \mathrm{~m}$

To complete the investigation, the parameter $C_{A r}$ used in Eq. (23) and Eq. (24) was evaluated. Based on the measured data of smooth and rough bed flows in literature, the quadratic equation (28) below is proposed for the representation of $C_{A r}$.

$C_{A r}=c_{1}\left(\xi_{d i p}\right)^{2}+c_{2}\left(\xi_{d i p}\right)+c_{3}$

where the empirical constants were found to be $c_{1}=5.0, c_{2}=1.0$ and $c_{3}=-1.0$. These empirical constants were found by the best-fitting approach from various measured data used in this study. A similar sigmoid model suggested by Bonakdari et al. (2008) was used to compute $\xi$ at velocity dip position, $\xi_{\text {dip }}$, since it is believed that the dip phenomenon was purely determined by $A_{r}$ of a flow.

$$
\xi_{\text {dip }}=\frac{k_{1}+k_{2}\left(A_{r}\right)^{k_{3}}}{k_{4}+\left(A_{r}\right)^{k_{3}}}
$$

The constants in equation (29) were found to be $k_{1}=40.1, k_{2}=1.0, k_{3}=4.4$ and $k_{4}=80.5$ by the best-fitting approach from the used data in this study; and they compared well with the suggested values by Bonakdari et al. (2008). The combined Eq. (28) and Eq. (29) effectively relate the determination of $C_{A r}$ to the occurrence of velocity dip. It shows that the proposed law fully relates all its parameters towards the flow properties; and without a doubt, it gives more confident to the finding of the proposed law since none of its parameters are determined randomly.

In Fig. 15, one could observe that all empirically estimated $C_{A r}$ correspond well with Eq. (28). However, the measurements by Coleman (1986) showed some discrepancies from the perfect line, in which the proposed calculation by Eq. (28) overpredicted the empirical finding. Numerically, the high water depth in the smooth bed measurements by Coleman (1986) gave a large denominator to $\xi_{\text {dip }}$ in Eq.

(29). Hence, a higher order of Eq. (28) for $C_{A r}$ might be needed to fully represent the smooth bed flow with high water depth. We found that by adding an extra degree to Eq. (28) as follows in Eq. (30), the norm error of Coleman (1986) measurements estimation could be reduced from an average $68.6 \%$ to only $4.1 \%$, which supports the previous statement.

$$
C_{A r}=c_{o}\left(\xi_{d i p}\right)^{3}+c_{1}\left(\xi_{d i p}\right)^{2}+c_{2}\left(\xi_{d i p}\right)+c_{3}
$$

where $c_{o}=-6.0$ as obtained from the best-fitting approach by various measured data used in this study. In Larrarte (2006) field measurements, the scattered data was observed when the calculated $C_{A r}$ was compared to the empirical finding. It is no doubt to be caused by the uncontrolled environment in the field study that gave some random behaviour to $C_{A r}$ empirically.

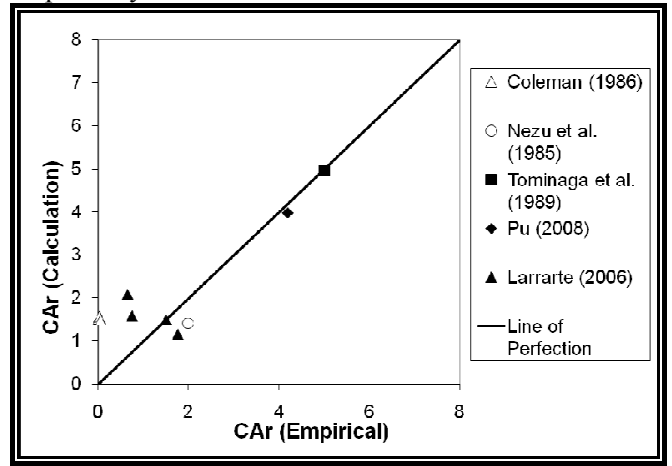

Fig. 15. Comparison of $C_{A r}$ calculated by Eq. (28) and by empirical fitting 


\section{CONClusion}

A novel law to estimate the secondary current induced velocity profile was proposed in this study to represent the velocity profile with: 1) heavy influence from the secondary current (by modifying the Reynolds stress distribution considered); and 2) universal representation for both smooth and rough channel flows. It is representative to any steady, uniform and fully developed turbulent open channel flow. From this study, the conclusions below could be drawn:

1. The proposed law was derived to concentrate in representing the velocity profile at the outer region of velocity distribution. It has been mathematically proven for all its simplifications and assumptions.

2. Due to the fact that the proposed law was derived directly from the Navier-Stokes momentum equation, it, unlike any modified log-wake law, could be used to express any velocity profile regardless the bed roughness condition. Hence in this study, a universal expression was fitted to solve both smooth and rough bed flows for this proposed law. In the universal law, different boundary considerations were used to represent smooth and rough bed conditions.

3. After applying the proposed law into various smooth and rough bed flow measurements from literature, we could conclude that it is capable to estimate both rough and smooth bed roughness conditions consistently. It is also found to be precisely representing the macro-rough bed flow velocity profile existing at the actual river flow.

4. The proposed law was derived to represent the velocity distribution of a flow with velocity dip phenomenon. Its comparison with the narrow channel flow application shows that it estimates the velocity dip well. After comparing the proposed law with Yang et al. (2004) law and the wake law in predicting the velocity dip, we found that the proposed law has improved the discrepancy in representing the measured data by an average of $43 \%$ compared to Yang et al. law, and by an average of $65 \%$ compared to the wake law.

A comparative test has been conducted for $C_{A r}$ parameter used in Eq. (23) to Eq. (24). The comparison was done between the empirically fitted data and the calculation estimated using Eq. (28). The comparison shows that $C_{A r}$ could be relatively well represented by a single Eq. (28).

\section{ACKNOWLEDGEMENTS}

The author acknowledges the cooperation and data from Dr H. Bonakdari and Dr F. Larrarte for this work during his research period at the French Institute of Science and Technology for Transport, Development and Networks (IFSTTAR), or previously known as the French Central Laboratory of Roads and Bridges (LCPC).

\section{REFERENCES}

Anwar, H. O. and R. Atkins (1980). Turbulence measurements in simulated tidal flow. Journal of the Hydraulics Division, 106(8), 1273-1289.

Bonakdari, H. (2006). Modélisation des écoulements en collecteurs d'assainissement - Application à la conception de points de mesures. $\mathrm{PhD}$ Thesis, L’Université de Caen, France. (In French)

Bonakdari, H., F. Larrarte, L. Lassabatere and C. Joannis (2008). Turbulent velocity profile in fullydeveloped open channel flows. Environmental Fluid Mechanics, 8(1), 1-17.

Cardoso, A. H., W. H. Graf and G. Gust (1989) Uniform flow in a smooth open channel. Journal of Hydraulic Research, 27(5), 603-616.

Coleman, N. L. (1986). Effects of suspended sediment on the open-channel velocity distribution. Water Resources Research, 22(10), 1377-1384.

Coles, D. (1956). The Law of the wake in the turbulent boundary layer. Journal of Fluid Mechanics, 1(2), 191-226.

Dey, S. and R. V. Raikar (2007). Characteristics of loose rough boundary streams at near-threshold. Journal of Hydraulic Engineering, 133(3), 288-304.

Franca, M. J. (2005). A field study of turbulent flows in shallow gravel-bed rivers. $\mathrm{PhD}$ Thesis, École Polytechnique Fédérale De Lausanne, Swissland.

Franca, M. J., R. M. L. Ferreira and U. Lemmin (2008). Parameterization of the logarithmic layer of doubleaveraged streamwise velocity profiles in gravel-bed river flows. Advances in Water Resource, 31(6), 915-925.

Gordon, L. (1992). Mississippi river discharge. RD Instrument, San Diego, CA.

Guo, J. and P. Y. Julien (2008). Application of the modified log-wake law in open-channels. Journal of Applied Fluid Mechanics, 1(2), 17-23.

Guo, J. and P. Y. Julien (2003). Modified log-wake law for turbulent flow in smooth pipes. Journal of Hydraulic Research, 41(5), 493-501.

Hurther, D. and U. Lemmin (2001). Discussion equilibrium near-bed concentration of suspended sediment. Journal of Hydraulic Engineering, 127(5), 430-433.

Ikeda, S. (1981). Self-formed straight channels in sandy beds. Journal of Hydraulic Research, 107(HY4), 389-406.

Keulegan, G. H. (1938). Laws of turbulent flow in open channels. Research Paper RP1151, J Res Nat Bur Stand, 121(6): 707-741. 
Kironoto B. A. and W. H. Graf (1994). Turbulence characteristics in rough uniform open-channel flow. Proceedings of the ICE - Water Maritime and Energy, 106(4), 333-344.

Larrarte, F. (2006). Velocity fields within sewers: An experimental study. Flow measurement and instrumentation, 17(5), 282-290.

Liu, Y. and H. Ni (2007). Modified log-wake Laws for turbulent flow of the outer and inner regions in smooth pipes. Journal of Hydrodynamics, Ser. B, 19(2), 188-194.

Mellor, G. L. and D. M. Gibson (1966). Equilibrium turbulent boundary layers. Journal of Fluid Mechanics, 24(2), 225-253.

Nezu, I. and R. Azuma (2004). Turbulence characteristics and interaction between particles and fluid in particle-laden open channel flows. Journal of Hydraulic Engineering, 130(10), 988-1001.

Nezu, I. and H. Nakagawa (1993). Turbulent openchannel flows. IAHR Monograph, A. A. Balkema, Rotterdam, the Netherlands.

Nezu, I., H. Nakagawa and W. Rodi (1985). Significant difference between secondary currents in closed channels and narrow open channels. Proceedings of 23rd IAHR Congress, Delft, The Netherlands, Vol. A: $125-132$.

Nikuradse, J. (1950). Laws of flow in rough pipes. (Translation in National Advisory Committee for Aeronautics), Technical Memorandum 1292, NACA, Washington, $62 \mathrm{p}$.

$\mathrm{Pu}$, J. H. (2008). Efficient Finite Volume Numerical Modelling and experimental study of $2 D$ shallow water free surface turbulent flows. $\mathrm{PhD}$ Dissertation, University of Bradford, UK.

$\mathrm{Pu}, \mathrm{J} . \mathrm{H}, \mathrm{H}$. Bonakdari, L. Lassabatere, C. Joannis and F. Larrarte (2010). Turbulent velocity profiles: A new law for narrow channels. La Houille Blanche International Journal, 3: 65-70.

Rodi, W. (1993). Turbulence models and their application in Hydraulics - a State of Art Review. $3^{\text {rd }}$ Ed., A. A. Belkema, Rotterdam.
Sarma, K. V. N., P. Lakshminarayana and N. S. L. Rao (1983). Velocity distribution in smooth rectangular open channels. Journal of Hydraulic Engineering, 109(2), 270-289.

Song, T. and W. H. Graf (1996). Velocity and turbulence distribution in unsteady open-channel flows. Journal of Hydraulic Engineering, 122(3), 141-154.

Song, T., W. H. Graf and U. Lemmin (1994). Uniform flow in open channels with movable gravel bed. Journal of Hydraulic Research, 32(6), 149-173.

Storm, K. B. and A. N. Papanicolaou (2007). ADV Measurements around a cluster microform in a shallow mountain stream. Journal of Hydraulic Engineering, 133(12), 1379-1389.

Termini, D. and M. Greco (2006). Computation of flow velocity in rough channels. Journal of Hydraulic Research, 44(6), 777-784.

Tominaga, A., I. Nezu, K. Ezaki, H. Nakagawa (1989). Three dimensional turbulent structure in straight open channel flows. Journal of Hydraulic Research, 27(1), 149-173.

Wang, Z. Q. and N. S. Cheng (2005). Secondary flows over artificial bed strips. Advances in Water Resource, 28(5), 441-450.

Wang, Z. Q. and N. S. Cheng (2006). Time-mean structure of secondary flows in open channel with longitudinal bedforms. Advances in Water Resource, 29(11), 1634-1649.

Yang, S. Q., S. Y. Lim and J. A. McCorquodale (2005). Investigation of near wall velocity in 3-D smooth channel flows. Journal of Hydraulic Research, 43(2), 149-157.

Yang, S. Q., S. K. Tan and S. Y. Lim (2004). Velocity distribution and dip-phenomenon in smooth uniform open channel flows. Journal of Hydraulic Engineering, 130(12), 1179-1186.

Yang, S. Q., W. L. Xu and G. L. Yu (2006). Velocity distribution in a gradually accelerating free surface flow. Advances in Water Resource, 29(12), 19691980. 